\title{
Respostas Glicêmicas após Exercício Isométrico com Handgrip em Idosas Portadoras de Diabetes Mellitus Tipo 2: Ensaio Clínico Randomizado
}

\section{Glycemic Responses After Isometric Exercise with Handgrip in Elderly Women with Type 2 Diabetes Mellitus: Randomized Clinical Trial}

\author{
Theo Victor Alves Soares do Rêgo ${ }^{1}$ \\ Thiago Borges Madureira Sabino ${ }^{2}$ \\ Nathália de Souza Melo Abath Barbosa ${ }^{3}$ \\ Maria Elizabeth Queiroz Holanda do Nascimento ${ }^{3}$ \\ Ana Beatriz de Carvalho Paz de Andrade ${ }^{3}$ \\ Elizabelle Pereira Costa ${ }^{3}$ \\ Jonathan Nicolas Ribeiro ${ }^{4}$ \\ Denise Maria Martins Vancea ${ }^{5}$
}

\section{RESUMO}

Objetivo: Avaliar o efeito de uma única sessão de exercício isométrico com handgrip sobre as respostas glicêmicas de idosas portadoras diabetes mellitus tipo 2 (DM2). Metodologia: Trata-se de um ensaio clínico randomizado. A amostra foi composta por 14 idosas portadoras de DM2, fisicamente ativas, com idade de $66,29 \pm 7,3$ anos. As participantes foram divididas aleatoriamente em dois grupos, o grupo controle (GC $n=7)$ e o grupo intervenção $(G I n=7)$. Com um dinamômetro de preensão manual, a força de contração voluntária máxima (CVM) foi medida em ambas as mãos. Para a intensidade da sessão experimental, foi utilizada $50 \%$ da carga de CVM encontrada no teste. A glicemia capilar foi coletada nos tempos pré, imediatamente após (IA), 30 e 60 minutos após a intervenção. Resultados: Não ocorreu diferença estatística entre as interações (grupo vs. tempo) e entre os grupos. Apenas o Gl apresentou diferença significativa em relação ao tempo (pré = $165.5 \pm 39.4 \mathrm{mg} / \mathrm{dL}$ vs. $I A=139.2 \pm 25.9 \mathrm{mg} / \mathrm{dL} ; p=0,026 ;$ pré $=$ $165.5 \pm 39.4 \mathrm{mg} / \mathrm{dL}$ vs. pós $30=126.2 \pm 17.1 \mathrm{mg} / \mathrm{dL} ; p=0,012$; pré $=165.5 \pm 39.4 \mathrm{mg} / \mathrm{dL}$ vs. pós $60=120.8 \pm 20.2 \mathrm{mg} / \mathrm{dL} ; p=$ $0,009)$ quando comparado como o GC. Conclusão: O exercício isométrico agudo com handgrip a $50 \%$ da CVM foi capaz de promover uma redução significativa nos níveis de glicemia capilar em idosas com DM2, expostas ao protocolo de exercício.

\section{DESCRITORES}

Exercício. Isométrico. Diabetes. Glicemia.

\begin{abstract}
Objective: To evaluate the effect of a single isometric exercise session with a handgrip on the glycemic responses of elderly women with type 2 diabetes mellitus (DM2). Methodology: This is a randomized clinical trial. The sample consisted of 14 elderly women with DM2, physically active, aged $66.29 \pm 7.3$ years. The participants were randomly divided into two groups, the control group (CG $n=7$ ) and the intervention group (IG $n$ $=7$ ). With a handgrip dynamometer, the maximum voluntary contraction force (CVM) was measured in both hands. For the intensity of the experimental session, $50 \%$ of the CVM load found in the test was used. Capillary blood glucose was collected in the pre-times, immediately after (AI), 30, and 60 minutes after the intervention. Results: There was no statistical difference between interactions (group vs. time) and between groups. Only the IG showed a significant difference in relation to time (pre $=165.5 \pm 39.4 \mathrm{mg} / \mathrm{dL}$ vs. Al $=139.2 \pm 25.9 \mathrm{mg} /$ $\mathrm{dL} ; \mathrm{p}=0.026 ;$ pre $=165.5 \pm 39.4 \mathrm{mg} / \mathrm{dL}$ vs. post $30=126.2$ $\pm 17.1 \mathrm{mg} / \mathrm{dL} ; \mathrm{p}=0.012$; pre $=165.5 \pm 39.4 \mathrm{mg} / \mathrm{dL}$ vs. post $60=120.8 \pm 20.2 \mathrm{mg} / \mathrm{dL} ; \mathrm{p}=0.009$ ) when compared with the CG. Conclusion: Acute isometric exercise with a handgrip at $50 \%$ of CVM was able to promote a significant reduction in capillary glycemia levels in elderly women with DM2, exposed to the exercise protocol.
\end{abstract}

\section{DESCRIPTORS}

Exercise. Isometric. Diabetes. Blood glucose.

${ }^{1}$ Educador Físico, membro do Grupo de Pesquisa Exercício Físico e Doenças Crônicas Não Transmissíveis - Universidade de Pernambuco/UPE, Recife, Pernambuco, Brasil.

${ }^{2}$ Discente do Programa de Pós-Graduação em Educação Física/PPGEF da Universidade Federal de Pernambuco/UFPE, Recife, Pernambuco, Brasil.

${ }^{3}$ Discente do Curso de Educação Física (Bacharelado) da Escola Superior de Educação Física/ESEF, da Universidade de Pernambuco - UPE, Recife, Pernambuco, Brasil.

${ }^{4}$ Discente do Programa de Pós-Graduação do Instituto de Ciências Biológicas - ICB da Universidade de Pernambuco -

UPE, Recife, Pernambuco, Brasil.

${ }^{5}$ Educadora Física, Professora Adjunta da Universidade de Pernambuco - UPE, Recife, Pernambuco, Brasil. 


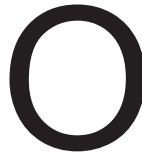
diabetes mellitus tipo 2 (DM2) é uma condição clínica multifatorial caracterizada por níveis elevados e sustentados da glicose sanguínea, em decorrência da resistência dos tecidos periféricos à ação da insulina ${ }^{1,2,3}$. O DM2 corresponde a cerca de $90 \%$ dos casos de diabetes mellitus (DM). Esse distúrbio metabólico é um dos principais fatores de risco para o desenvolvimento de complicações microvasculares e macrovasculares na população idosa, sobretudo pelo risco de desenvolver doença arterial coronariana (DAC), doença arterial periférica (DAP), doença cerebrovascular e disfunção endotelial ${ }^{3-7}$.

$$
\text { É consenso que estratégias }
$$
não farmacológicas, como mudanças no comportamento alimentar e a adoção da prática regular de atividade física, devem fazer parte do tratamento e controle do DM2 $2^{2,3,7,8}$. Nesse sentido, o exercício físico é fortemente recomendado para os indivíduos diabéticos do tipo 2, por ser capaz de melhorar a resistência à insulina, controlar o peso corporal, auxiliar no tratamento de problemas psicossociais e, em longo prazo, reduzir os riscos de desenvolver doenças cardiovasculares e cerebrovasculares, consideradas as causas mais comuns de morte no paciente diabético $0^{9,10,11}$. O exercício físico também é capaz de aumentar, por meio da contração muscular, a expressão e tradução das proteínas transportadoras de glicose (GLUT) pela ativação de diferentes vias de sinalização molecular e, dentre elas, a ativação da via da proteína quinase ativada por AMPK (activated protein kinase), independentemente da modalidade do exercício $^{12,13}$.
Nesse sentido, o músculo esquelético desempenha um papel chave na manutenção e regulação da glicemia, sendo responsável por cerca de 70 a $90 \%$ da captação de glicose sanguínea em estado pós-prandial ${ }^{14}$. Esse aumento na captação de glicose acontece devido ao nível crescente da demanda metabólica imposta pelo exercício físico para geração de energia, exclusivamente, pela via glicolítica intramuscular ${ }^{14,15}$. As respostas glicêmicas após o exercício físico são de extrema relevância clínica para indivíduos idosos portadores de DM2, sobretudo, pelos benefícios de manter os níveis de glicose sanguínea controlados.

A força isométrica de preensão manual, avaliada com handgrip, tem sido associada a uma variedade de doenças crônicas não transmissíveis (DCNTs), como o DM2, e morte prematura em idosos ${ }^{16}$. Esses indivíduos geralmente apresentam fraqueza muscular e baixa capacidade de geração de força de preensão manual. O treinamento isométrico com handgrip tem sido utilizado como uma estratégia alternativa de baixo custo para o tratamento e a prevenção de doenças cardiovasculares e metabólicas em idosos $^{16}$.

Em uma revisão sistemática foi evidenciado benefícios cardiovasculares, como a diminuição da pressão arterial (PA), após exercícios envolvendo pequenos grupos musculares, especificamente, o exercício isométrico realizado com handgrip ${ }^{17}$. Apesar de existir fortes evidências dos efeitos desses exercícios sobre as respostas cardiovasculares em idosos, os mecanismos ainda não estão claros sem relação aos efeitos desse tipo de treinamento sobre as respostas glicêmicas 
agudas em indivíduos idosos portadores de DM2. Portanto, é de fundamental importância investigar a influência desse tipo de exercício sobre as respostas glicêmicas, contribuindo para o controle da doença e em pesquisas futuras.

Sendo assim, o objetivo deste estudo foi avaliar o efeito agudo do exercício isométrico com handgrip sobre as respostas glicêmicas de idosas portadoras de DM2.

\section{METODOLOGIA}

\section{Participantes}

Participaram do estudo 14 (quatorze) idosas portadoras de diabetes mellitus tipo 2, com média de idade de 66,29 $\pm 7,3$ anos, fisicamente ativas e familiarizadas com o uso do handgrip. A amostra foi não probabilística, por conveniência, compostas por participantes de um programa de exercício físico supervisionado para diabéticos de uma Universidade Pública do Estado de Pernambuco. O tamanho amostral foi determinado por meio do software $\mathrm{G}^{*} \mathrm{Power}$ 3.118. Com base em uma análise a priori, foi calculado um " $\mathrm{n}$ " de 14 indivíduos, após a adoção de um poder observado $\beta$ de 0,80 , nível de significância $\alpha=0,05$, coeficiente de correlação entre as medidas repetidas de 0,5 , correção de não esfericidade de 1 e tamanho de efeito de 0,34 . Verificou-se que os 14 sujeitos foram suficientes para fornecer $83,2 \%$ do poder estatístico. Para o cálculo amostral, foram empregados os procedimentos sugeridos por Beck ${ }^{19}$.

Trata-se de um ensaio clínico randomizado controlado (ECR), cujos participantes foram separados, de forma simples em dois grupos: grupo controle (GC, n=7) e grupo intervenção ( $\mathrm{GI}, \mathrm{n}=7$ ). Os critérios de inclusão foram: serem mulheres idosas, fisicamente ativas e diagnosticadas com DM2; enquanto os de exclusão foram: possuírem algum problema osteomioarticular ou qualquer contraindicação médica que as impedissem de realizar ou deixar de participar de qualquer procedimento dos protocolos experimentais. Após serem informados sobre os riscos e os benefícios da pesquisa, os sujeitos assinaram o Termo de Consentimento Livre Esclarecido (TCLE), elaborado de acordo com a Declaração de Helsinque e Resolução $n^{\circ}$. 466/2012, do Conselho Nacional de Saúde (CNS). O estudo foi aprovado pelo Comitê de Ética local (Protocolo CEP/UPE: $n^{\circ}$ 007/09). O diagrama de fluxo da pesquisa está representado na (Figura 1).

Procedimentos

Os participantes receberam orientações que deveriam ser seguidas nas 24 horas antes das sessões experimentais, como manter sua rotina normal de alimentação e o horário de sono, abster-se de qualquer prática de exercício físico, não ingerir bebidas alcoólicas e manter as medicações usuais de controle glicêmico. Durante a primeira e a última visita ao Laboratório de Biodinâmica, foram realizados os seguintes procedimentos: inicialmente, anamnese clínica, medidas antropométricas (peso e estatura), bem como medida da glicemia, garantindo a inclusão e a elegibilidade do estudo; também foram coletados os dados demográficos e uso de medicamentos. Foram realizadas, 48 horas após, medições de glicemia capilar 
Figura 1. Diagrama de fluxo do Estudo ${ }^{20}$.

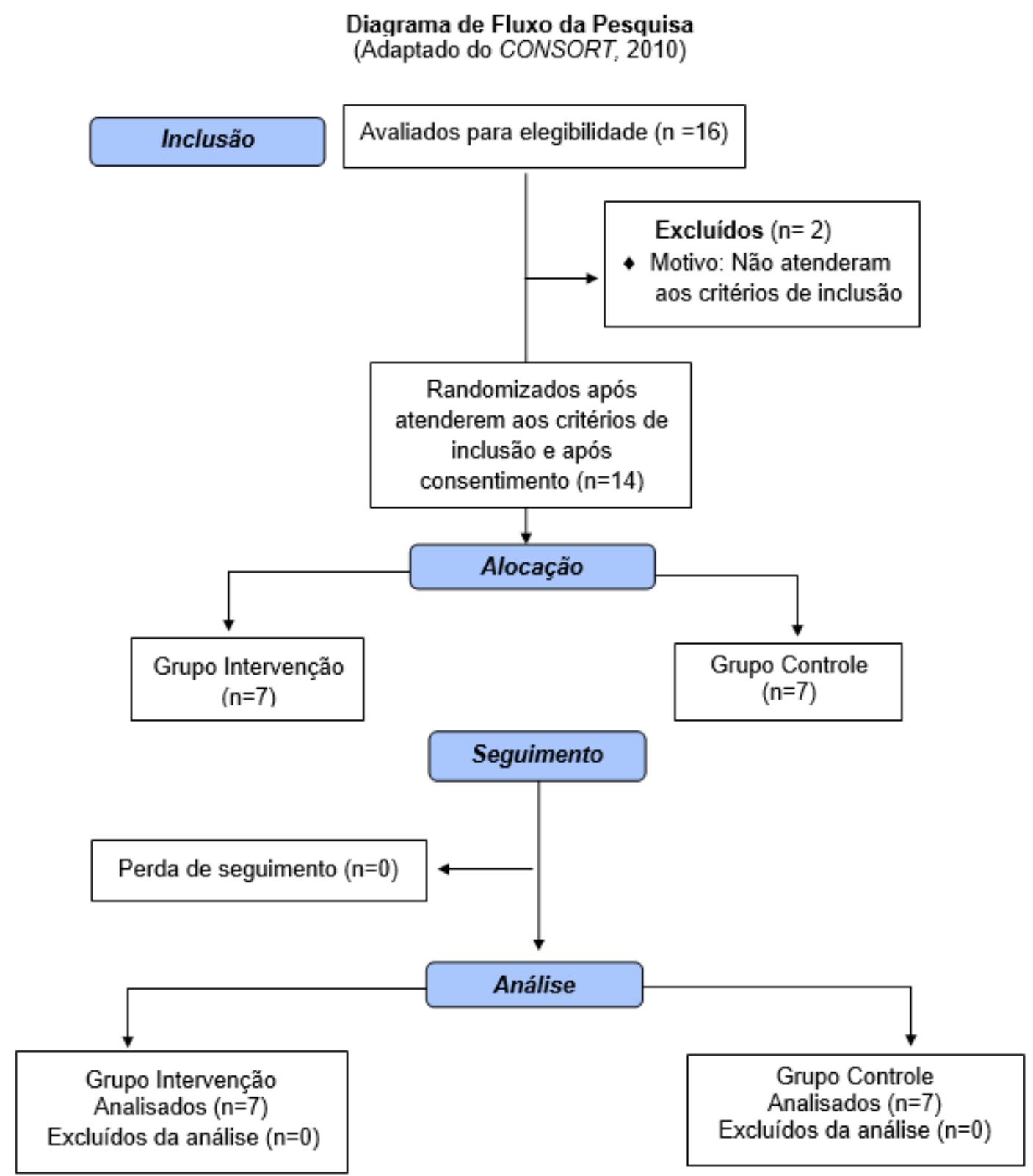

$\mathrm{MC}=$ massa corporal; $\mathrm{El}=$ exercício isométrico; $\mathrm{CVM}=$ contração voluntária máxima .

e testes de contração voluntária máxima (CVM); em seguida, foi determinada a carga de treinamento por meio de teste de CVM e realizada uma única sessão de exercício isométrico com handgrip a $50 \%$ da carga encontrada no teste de CVM (Figura 2). Todos os procedimentos foram realizados no mesmo local, horário e período do dia.

Avaliação Antropométrica e Glicemia Capilar Inicialmente, foi utilizada uma balança 
Figura 2. Desenho experimental.

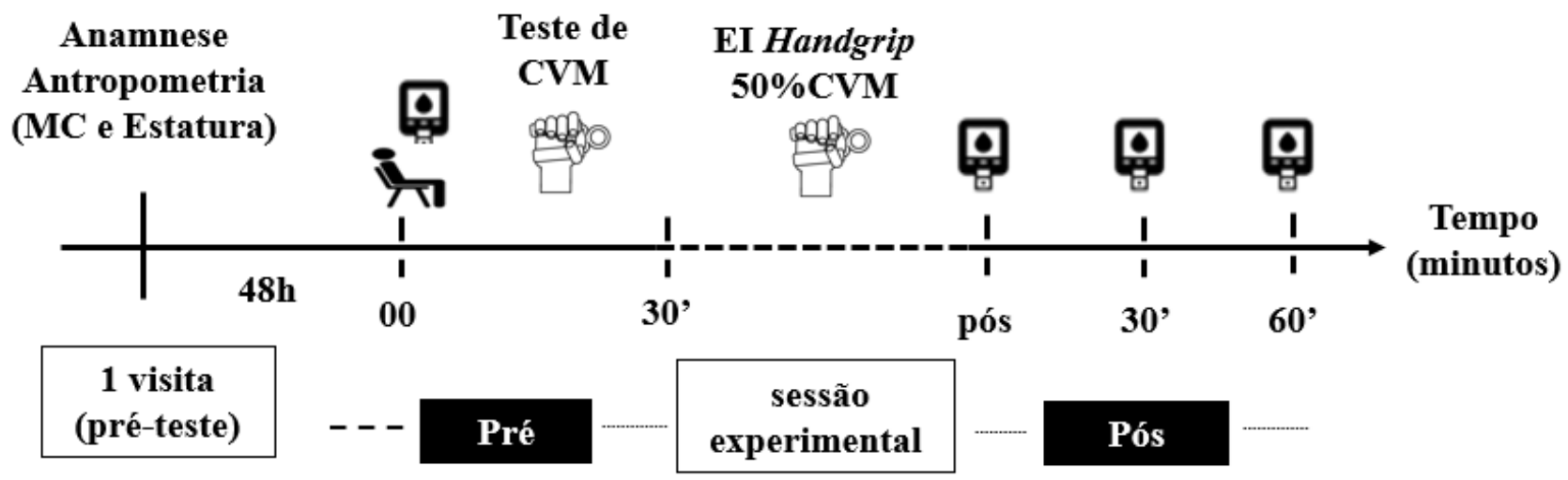

digital (Filizola, Brasil) com escala de precisão $0,1 \mathrm{~kg}$, devidamente calibrada para avaliação da massa corporal. Em seguida, a estatura foi avaliada por meio de estadiometro de parede (Sanny, Brasil) com precisão de 0,05m. Essas medidas foram utilizadas, posteriormente, para obtenção do índice de massa corporal (IMC) em m $\mathrm{em}^{2} / \mathrm{kg}^{-1}$.
A glicemia capilar foi avaliada por meio de glicosímetro portátil (G-Tech modelo Free Lite $\left.{ }^{\circledR}\right)$ antes, imediatamente após, 30 e 60 minutos após a realização do protocolo experimental. A coleta do sangue capilar foi realizada sempre nos dedos mínimos ou anelares, descartando a primeira gota, em seguida, utilizada a segunda gota para análise ${ }^{21}$.

Tabela 1. Características dos sujeitos da amostra

\begin{tabular}{|c|c|c|c|c|c|}
\hline & \multicolumn{2}{|c|}{$\mathrm{GI}(\mathrm{n}=7)$} & \multicolumn{2}{|c|}{$\mathrm{GC}=(\mathrm{n}=7)$} & \multirow[b]{2}{*}{$p$} \\
\hline & Média \pm DP & IC95\% & Média \pm DP & IC95\% & \\
\hline Idade (anos) & $65.0 \pm 6.2$ & $(59.25-70.75)$ & $67.5 \pm 8.6$ & $(59.58-75.56)$ & 0.535 \\
\hline \multirow{3}{*}{$\begin{array}{l}\text { MC }(\mathrm{kg}) \\
\text { Estatura } \\
(\mathrm{cm}) \\
\mathrm{IMC}\left(\mathrm{kg} \cdot \mathrm{m}^{2}\right)\end{array}$} & $67.0 \pm 9.2$ & $(58.44-75.56)$ & $56.1 \pm 10.2$ & $(46,67-65,61)$ & $0.059^{*}$ \\
\hline & $1.52 \pm 0.07$ & $(1.08-2.07)$ & $1.51 \pm 0.05$ & $(1,15-1.98)$ & 0.692 \\
\hline & $28.80 \pm 4.5$ & $(24.56-33.04)$ & $24.53 \pm 4.7$ & (20.14-28.92) & 0.113 \\
\hline $\operatorname{NAF}(\%)$ & 100 & - & 100 & - & - \\
\hline $\begin{array}{l}\text { Glicemia } \\
\text { (mg/dl) }\end{array}$ & $165.57 \pm 39$ & (129.06-202.08) & $143.71 \pm 28.3$ & $(117.48-169.95)$ & 0.257 \\
\hline \multicolumn{6}{|c|}{ Medicações em uso (\%) } \\
\hline $\mathrm{HO}$ & 57,1 & - & 57,1 & - & - \\
\hline \multirow{2}{*}{$\begin{array}{l}\text { Insulina } \\
\mathrm{HO}+ \\
\text { insulina }\end{array}$} & - & - & 42,9 & - & - \\
\hline & 42,9 & & - & & \\
\hline
\end{tabular}


Avaliação da Força Isométrica Máxima

A força de contração voluntária máxima (CVM) foi medida em ambas as mãos, iniciando-se pela não dominante, por meio do handgrip da Marca CAMRY, modelo EH 101 digital. Os sujeitos permaneceram sentados, com coluna ereta, o ombro posicionado em adução e rotação neutra, cotovelo flexionado a $90^{\circ}$, com o antebraço em posição pronada e punho neutro, podendo movimentá-lo até $30^{\circ}$ graus de extensão. Os sujeitos foram estimulados, verbalmente, a atingir o máximo desempenho. Foi utilizado o melhor resultado de 3 (três) tentativas para calcular a intensidade do exercício.

Protocolo de Exercício

Para a sessão experimental, foi utilizado $50 \%$ da carga máxima encontrada no teste de CVM, sendo aplicado o protocolo unilateral de quatro séries de cinco repetições, mantidos 10 segundos em contração isométrica e 20 segundos em recuperação, com intervalo de 60 segundos entre as séries ${ }^{22}$. Foi sugerido que os indivíduos concentrassem seus esforços em manter um nível de contração entre os intervalos de $\pm 1 \mathrm{~kg} / \mathrm{f}$ de $50 \%$ da CVM. Para o cálculo do volume total de trabalho, a carga foi multiplicada pelo número de séries e repetições completas na sessão do exercício (carga $x$ séries $x$ repetições).

Análise Estatística

Os dados foram analisados utilizando a estatística descritiva (média, desvio padrão, erro-padrão e intervalos de confiança). Os testes de Shapiro-Wilk e de Levene foram utilizados para verificar a normalidade e a homogeneidade da distribuição dos dados. De acordo com as análises, os dados foram considerados normais. Uma análise de variância (ANOVA) de medidas repetidas e o teste post-hoc de Bonferroni foram utilizados para identificação das diferenças entre as médias das interações grupo vs. tempo (GC e GI vs. imediatamente após, pós 30 e 60 minutos), dos grupos (GC vs. GI) e do tempo (imediatamente após, pós 30 e 60 minutos) para as variáveis de desfecho do estudo. O pressuposto de esfericidade foi analisado por meio do teste de Mauchly. A esfericidade não foi assumida e, dessa forma, foi adotada a correção de Greenhouse-Geiseer para análise das variâncias. Além disso, o tamanho do efeito foi calculado por meio do Eta squared $\left(\eta^{2}\right)$ para avaliar a magnitude da evidência e estabelecer diferenças para uma melhor aplicação prática. Os dados foram analisados pelo software SPSS 21 for Windows e o nível de significância assumido foi $p<0,05$.

\section{RESULTADOS}

$\mathrm{Na}$ análise comparativa dos efeitos do exercício isométrico com handgrip sobre a glicemia capilar, não ocoreu interações significativas entre grupo vs. tempo $(F=1,460$, $\left.\eta^{2}=0,199, p=0,090\right)$ e grupo $\left(F=0,077, \eta^{2}\right.$ $=0,006, p=0,786$ ) (Tabela 2). No entanto, o teste de post-hoc de Bonferroni mostrou que apenas o grupo intervenção apresentou diferença significativa em relação ao tempo [F $\left.(1,460,17,51)=12,712, \eta^{2}=0,514, p=0,001\right]$ (Tabela 3).

Nas Figuras 3, 4 e 5 são apresentados, respectivamente, os dados referentes aos 
Tabela 2. Análise comparativa das médias dos grupos, do delta de variação das diferenças e do tamanho do efeito da glicemia capilar nos momentos pré, imediatamente após, pós 30 e 60 minutos da sessão experimental

\begin{tabular}{cccccc}
\hline $\begin{array}{c}\text { Glicemia capilar } \\
(\mathrm{mg} / \mathrm{dl})\end{array}$ & $\mathrm{Gl}=(\mathrm{n}=7)$ & $\mathrm{GC}=(\mathrm{n}=7)$ & & & \\
\cline { 2 - 6 } & Média $\pm \mathrm{DP}$ & Média $\pm \mathrm{DP}$ & $\Delta \pm \mathrm{EP}$ & $P$ & $\begin{array}{c}\text { Eta }^{2} \\
\text { Parcial }\end{array}$ \\
\hline Pré & $165.5 \pm 39.4$ & $143.71 \pm 28.3$ & $21.8 \pm 18.3$ & 0.257 & 0.105 \\
Imediatamente após & $139.2 \pm 25.9$ & $135.2 \pm 20.2$ & $4.0 \pm 12.4$ & 0.753 & 0.009 \\
Pós 30 min & $126.2 \pm 17.1$ & $134.8 \pm 29.4$ & $8.5 \pm 12.8$ & 0.519 & 0.035 \\
Pós 60 min & $120.8 \pm 20.2$ & $124.00 \pm 27.1$ & $3.1 \pm 12.8$ & 0.810 & 0.005 \\
\hline
\end{tabular}

Resultados da análise de variância ANOVA one way com medidas repetidas. Os valores são apresentados em média \pm padrão; GI = grupo intervenção; GC = grupo controle; $\mathrm{mg} / \mathrm{dl}$ : miligramas por decilitro; $\Delta$ = delta de variação é apresentado em erro padrão; $p=$ nível de significância.

Tabela 3. Análise comparativa das diferenças médias absolutas dos efeitos da interação entre grupo e tempo sobre a glicemia capilar nos momentos pré, imediatamente após, pós 30 e 60 minutos da sessão experimental

\begin{tabular}{ccccc}
\hline \multirow{2}{*}{ Glicemia capilar $(\mathrm{mg} / \mathrm{dL})$} & \multicolumn{2}{c}{$\mathrm{GI}(\mathrm{n}=7)$} & \multicolumn{2}{c}{$\mathrm{GC}(\mathrm{n}=7)$} \\
\cline { 2 - 5 } & $\mathrm{DM} \pm \mathrm{EP}$ & $p$ & $\mathrm{DM} \pm \mathrm{EP}$ & $p$ \\
\hline Imediatamente após vs pré & $26,2 \pm 7.4$ & $0.026^{*}$ & $8.4 \pm 7.4$ & 1.000 \\
Pós 30 vs pré & $39.2 \pm 10.0$ & $0.012^{*}$ & $8,8 \pm 10.0$ & 1.000 \\
Pós 60 vs pré & $44.7 \pm 10.9$ & $0.009^{*}$ & $19,7 \pm 10.9$ & 0.586 \\
\hline
\end{tabular}

Os dados são apresentados em diferença média \pm erro padrão (DM $\pm \mathrm{EP})$. *Diferença estatisticamente significativa $(p<0,05)$. $\mathrm{GI}$ = grupo intervenção; $\mathrm{GC}$ = grupo controle; mg/dL: miligramas por decilitro.

Figura 3. Diferença média dos níveis de Glicemia capilar entre as interações (grupo vs. tempo) em resposta ao exercício isométrico com handgrip a 50\% da CVM

\section{(Grupo*Tempo)}

$$
(\mathrm{F}=1,460, \eta 2=0,199, p=0,090)
$$

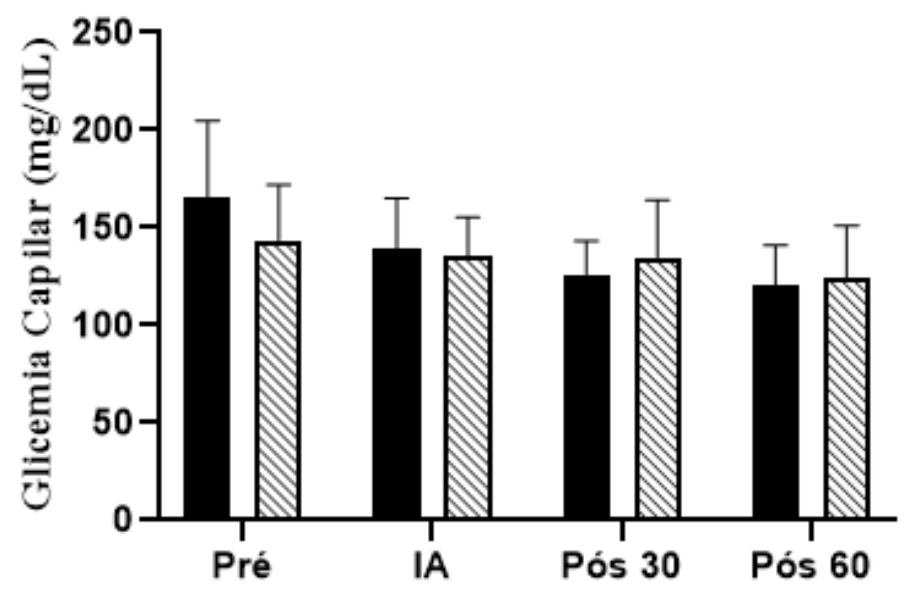

As barras pretas representam os valores do grupo intervenção (GI) e as listradas o grupo controle (GC) 
Figura 4. Diferença média absoluta dos níveis de Glicemia capilar intragrupos nos momentos pré, imediatamente após, pós 30 e 60 minutos após exercício isométrico com handgrip a 50\% da CVM.

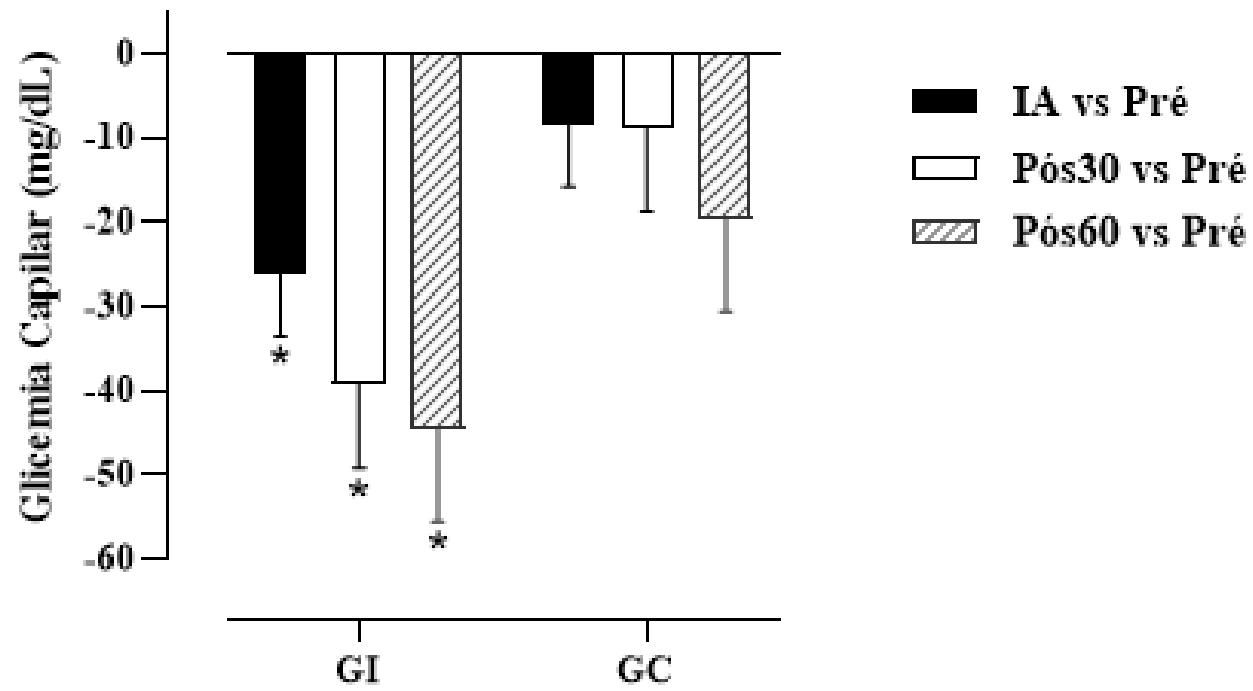

*Diferença significativa $(p<0,05)$. $G I$ = grupo intervenção; $G C=$ grupo controle.

Figura 5. Níveis absolutos de glicemia capilar dos sujeitos da amostra nos momentos pré, imediatamente após, pós 30 e 60 minutos da sessão experimental

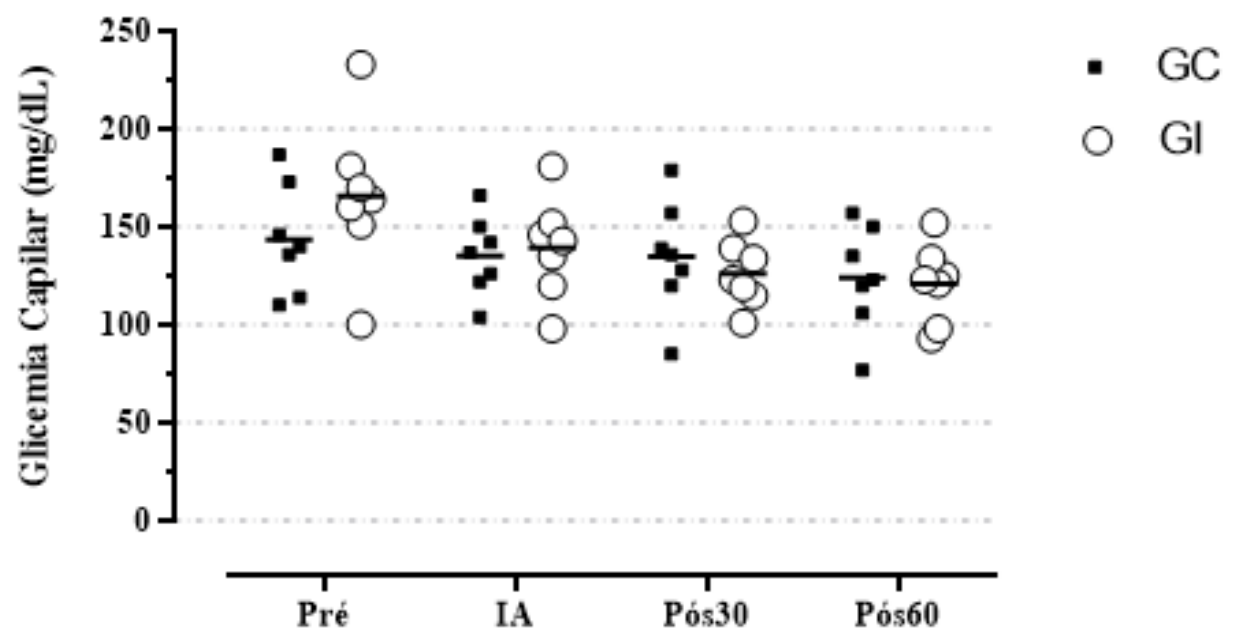


efeitos do exercício isométrico com handgrip sobre os níveis de glicemia capilar nas interações entre os grupos (intervenção e controle) vs. tempo (pré, imediatamente após, pós 30 e pós 60 minutos); a diferença média absoluta da redução da glicemia entre as interações grupo vs. tempo e os valores médios absolutos da glicemia de cada sujeito vs. tempo.

\section{DISCUSSÃO}

O presente ensaio clínico analisou o efeito agudo de uma única sessão de exercício isométrico com handgrip a 50\% da CVM sobre a glicemia capilar de mulheres idosas com DM2.

Conforme os resultados encontrados, não ocorreu diferença estatisticamente significante entre os grupos nos momentos analisados. Ambos os grupos apresentaram diminuição da glicemia, no entanto, apenas o grupo experimental apresentou redução significativa entre as interações dos momentos pré vs imediatamente após, pré vs pós 30 e 60 minutos após a sessão experimental. Para nosso conhecimento, esse é o primeiro estudo a analisar as respostas agudas da glicemia capilar após o exercício isométrico com handgrip a $50 \%$ da CVM em mulheres idosas com DM2.

Embora não tenham sido observadas diferenças estatisticamente significantes na glicemia capilar entre os grupos, os achados do presente estudo indicam que uma única sessão de exercício isométrico com handgrip a $50 \%$ da CVM é capaz de reduzir significativamente a glicemia capilar de idosas portadoras de DM2 expostas ao exercício. De acordo com as análises, a magnitude do tamanho do efeito da redução da glicemia capilar é biologicamente relevante $\left(\mathrm{F}=(1,460,17,51)=12,712, \eta^{2}=0,514, p=\right.$ $0,001]$, sobretudo pela redução do risco de complicações micro e macrovasculares, seja de forma aguda ou crônica. Do ponto de vista prático, essa redução é clinicamente importante para orientar os profissionais de saúde que participam de programas de controle e tratamento do DM2 em mulheres idosas portadoras da doença.

O presente estudo não teve como objetivo investigar os mecanismos fisiológicos pelos quais uma única sessão de exercício isométrico com handgrip a $50 \%$ da CVM promoveu maior redução na glicemia no grupo intervenção do que o grupo controle. Não obstante, sabe-se que o exercício isométrico promove contração muscular, que por sua vez ativa vias de sinalização moleculares independentes de insulina e consequentemente aumentam a captação de glicose circulante pelo músculo esquelético ${ }^{14}$. Em estudos com animais, 10 semanas de exercício isométrico ( $3 \mathrm{~min} / 3$ sessões/5 vezes por semana) diminuiu os níveis de glicose sanguínea em $\sim 30 \%$ após 2 horas $^{23}$. Entretanto, nosso estudo é o primeiro a investigar as respostas glicêmicas capilares com intensidade a 50\% da CVM em idosas com DM2.

Esses mecanismos, pelo menos em 
parte, podem explicar uma maior redução da glicemia capilar demonstrada no grupo intervenção quando comparada ao grupo controle. Assim, os nossos achados sugerem que realizar exercício isométrico com handgrip a 50\% da CVM é eficaz para reduzir agudamente a glicemia capilar de idosas diabéticas do tipo 2 com as características da amostra do nosso estudo.

É válido ressaltar, que existem algumas limitações no presente estudo, que devem ser levadas em consideração na interpretação dos resultados obtidos: 1) a amostra era composta por idosas diabéticas do tipo 2 que faziam uso de insulina isoladamente ou associado com fármacos hipoglicemiantes orais de diferentes princípios ativos e dosagem; 2) não foi analisado o tempo de diagnóstico da doença; 3) o tempo de análise das respostas da glicemia capilar foi de até 60 minutos após o exercício físico, o que torna-se necessário estudos que consigam prolongar o tempo de análise para pelo menos 24 horas, por exemplo; 4) o teste de CVM foi realizada no mesmo dia da sessão experimental, isso talvez possa ter alterado o nível de glicemia inicial do grupo intervenção.

Por fim, embora a glicemia capilar seja considerada um dos parâmetros para o controle da glicemia, pode haver divergências em relação aos equipamentos de análise sanguínea, as fitas reagentes, o tempo de perfusão tecidual da glicose circulante, o estado emocional do paciente, as medicações utilizadas, o tempo da doença, os grupos musculares envolvidos, a intensidade, o volume e a duração do exercício. Portanto, os achados do presente estudo devem ser interpretados com cautela, devido às limitações metodológicas apresentadas.

Como aplicação prática, esse tipo de exercício é de simples aplicação, fácil compreensão, baixo custo aquisitivo, tem relevância clínica e deve ser utilizado no tratamento do DM2, especialmente para aqueles que mais sofrem com seus agravos à saúde e encontram-se impossibilitados de inserir-se em práticas de exercícios convencionais. Nesse contexto, os indivíduos de alto risco cardiovascular, aqueles com neuropatia periférica avançada e presença de ulcerações nos pés se beneficiarão dos exercícios isométricos com handgrip.

\section{CONCLUSÃO}

As repostas glicêmicas ao exercício isométrico com handgrip agudo, a $50 \%$ CVM, foi capaz de promover uma redução nos níveis de glicemia capilar em idosas do grupo intervenção expostas ao protocolo de exercício. Os desfechos são relevantes do ponto de vista clínico. Portanto, são necessários o desenvolvimento de pesquisas futuras com diferentes intensidades, com maior tempo de análise e programas crônicos de treinamento. 


\section{REFERÊNCIAS}

1. American College of Sports Medicine (ACSM). ACSM's guidelines for exercise testing and prescription. Lippincott Williams \& Wilkins; 2018.

2. American Diabetes Association (ADA), Standards of medical care in diabetes. J Clinical and Applied Research and Education. 2020; 43(1):S1-S212.

3. Sociedade Brasileira de Diabetes (SBD). Diretrizes da Sociedade Brasileira de Diabetes, p. 112-115. São Paulo: Editora Clannad; 2020.

4. Word Health Organization (WHO). The Top 10 Causes of Death.

5. LeeJH, LeeR, Hwang HM, Hamilton TM, Park Y. The effects of exercise on vascular endothelial function in type 2 diabetes: A systematic review and meta-analysis. Fred DiMenna. Diabetology and Metabolic Syndrome. 2018; 10(1):1-14.

6. Qiu S, Cai X, Yin H, Sun Z, Zügel M, Steinacker MJS, et al. Exercise training and endothelial function in patients with type 2 diabetes: a meta-analysis. Cardiovascular Diabetology. 2018; 17:64.

7. Internacional Diabetes Federation (IDF). IDF Diabetes Atlas, $9^{\text {th }}$ ed. Brussels, Belgium 2019.

8. Tavares M, Ferreira Neta J, França J, Ribeiro J, Barbosa $\mathrm{C}$, Silva V, et al. Análise da percepção de diabéticos tipo 2 sobre a doença e o tratamento. J Epidemiologia e Controle de Infecção.2016; 6(2):85-91.

9. Bally L, Laimer M, Stettler C. Exercise-associated glucose metabolism in individuals with type 1 diabetes mellitus. Current Opinion in Clinical Nutrition \& Metabolic Care. 2015; 18(4):428- 433.

10. Madsen SM, Thorup AC, Overgaard K, Jeppesen PB. High intensity interval training improves glycaemic control and pancreatic $\beta$ cell function of type 2 diabetes patients. PLoS ONE 2015; 10 (8): e0133286.

11. Benjamin EJ, Muntner P, Alonso A, Bittencourt SM, Callaway WC, Carson et al. Heart disease and stroke statistics-2019 update: a report from the American Heart Association. Circulation. 2019; 139(10):e56-e528.
12. Röhling M, Herder C, Stemper T, Müssig K. Influence of acute and chronic exercise on glucose uptake. Journal of Diabetes Research 2016; v. 2016:2868652.

13. Pacheco C, Dos Santos PHL, Alves OJ, De Queiroz NA, Soares MP, Ceccatto MV. Regulação gênica da via AMPK pelo exercício físico: revisão sistemática e análise in Silico. Rev Bra Medicina do Esporte. 2017; 23(4):328-334.

14. Evans LP, McMillin LS, Weyrauch AL, Witczak AC. Regulation of Skeletal Muscle Glucose Transport and Glucose Metabolism by Exercise Training. Nutrients. 2019; 11(10):2432.

15. McGee LS, Hargreaves M. Exercise adaptations: molecular mechanisms and potential targets for therapeutic benefit. Nature Reviews Endocrinology. 2020; 16(9):495-505.

16. McGrath R, Johnson N, Klawitter L, Mahoney S, Trautman $\mathrm{K}$, Carlson $\mathrm{C}$, et al. What are the association patterns between handgrip strength and adverse health conditions? A topical review. SAGE Open Medicine.2020; (8):1-12.

17. Farah QB, Germano-Soares HA, Rodrigues CLS, Santos $X C$, Barbosa SS, Viana $C L$, et al. Acute and chronic effects of isometric handgrip exercise on cardiovascular variables in hypertensive patients: a systematic review. Sports. 2017; 5(3):55.

18. Faul F, Erdfelder E, Lang GA, Buchner A. G* Power 3: A flexible statistical power analysis program for the social, behavioral, and biomedical sciences. Behavior Research Methods. 2007; 39(2):175-191.

19. Beck TW. The importance of a priori sample size estimation in strength and conditioning research. Journal of Strength and Conditioning Research. 2013; 27(8): 2323-2337.

20. CONSORT. The CONSORT 2010 Flow Diagram. Consort Transparent Reporting of Trials 2019.

21. Hortensius J, Slingerland JR, Kleefstra N, Logtenberg JJS, Groenier HK, Houwelling et al. Self-monitoring of blood glucose: the use of the first or the second drop of blood. Diabetes Care. 2011; 34(3):556-560. 
22. Olher RDRV, Bocalini SD, Bacurau FR, Rodriguez D, Figueira Jr A, Pontes Jr, et al. Isometric handgrip does not elicit cardiovascular overload or post-exercise hypotension in hypertensive older women. Clinical interventions in aging. 2013; (8): 649, 2013.

23. Krüger K, Gessner DK, Seimetz M, Banisch J, Ringseis $\mathrm{R}$, Eder $\mathrm{K}$, et al. Functional and Muscular Adaptations in an Experimental Model for Isometric Strength Training in Mice. PLoS ONE. 2013; 8(11).

\section{CORRESPONDÊNCIA}

Thiago Borges Madureira Sabino

Av. Prof. Moraes Rego, 1235

Cidade Universitária, Universidade Federal de Pernambuco, Recife - PE,

Brasil, CEP: 50670-901.

E-mail: thiago_madureira@hotmail.com 